\title{
Chemical Homogeneity in Entropy-Stabilized Complex Metal Oxides
}

\author{
Ali Moballegh, Christina M. Rost, Jon-Paul Maria, Elizabeth C. Dickey
}

Department of Materials Science and Engineering, North Carolina State University, Raleigh, NC, USA

Innovation in new mixtures of constituents can lead to discover exciting new materials with unexpected properties and revolutionary applications $[1,2]$. It is known, the Gibbs energy needs to be minimized, as the main requirement, to achieve a stable single phase compound. Conventional approach to minimize the total energy of system is searching for a large and negative enthalpy. However, in this work, we show that the phase stability can be reached where the configurational entropy is maximized with mixing as many diverse elements as possible.

In this work, five binary metal oxides, $\mathrm{MgO}, \mathrm{CaO}, \mathrm{NiO}, \mathrm{CuO}$, and $\mathrm{ZnO}$, were chosen considering favored coordination, ionic radii, and diversity in crystal structures. An equimolar mixture of the constituent metaloxide powders were mixed and pressed into ceramic pellets. The pellets were subsequently annealed in an air until equilibrium was achieved and then quenched to the room temperature. A series of samples was prepared and annealed at temperatures ranging from $700^{\circ} \mathrm{C}$ to $1100^{\circ} \mathrm{C}$ to monitor the phase evolution. In Figure 1, X-ray diffraction (XRD) patterns show two distinguishable phases, rocksalt and wurtzite, are present up to $700^{\circ} \mathrm{C}$, but are fully converted to single-phase rocksalt between $850^{\circ} \mathrm{C}$ and $900^{\circ} \mathrm{C}$. The multiphase state reappears when the fully uniform single-phase sample was reannealed at $700^{\circ} \mathrm{C}$ for 2 hours, which is consistent with an entropy-driven mechanism.

Microstructure and microchemistry of the phase-pure sample was studied by using a variety of electron microscopy and spectroscopy techniques. An Allied Multiprep polishing system was utilized to prepare a cross-sectional electron microscopy sample by wedge polishing technique [3]. An aberration corrected FEI Titan G2 60-300 kV S/TEM equipped with an X-FEG source and an advanced Super-XTM EDS detector system was used to analyze the structure and chemistry of the sample. The microscope was operated at $200 \mathrm{kV}$ for high-angle annular dark-field (HAADF) scanning transmission electron microscope (STEM) imaging and energy dispersive x-ray spectroscopy (EDS) mapping with a convergence semi-angle of $15 \mathrm{mrad}$. STEM and EDS maps obtained at a variety of length scales reveal chemical and structural homogeneity of the cation mixture within the sample.

To further understand the phase stability and decomposition, the XRD studies (Fig. 1) are reproduced via in-situ heating experiments performed in Protochips Adoro ${ }^{\mathrm{TM}}$ heating holder in a JEOL 2010F operated at $200 \mathrm{kV}$. The phase evolution mechanisms and kinetics are studied by a combination of energy-filtered TEM, HAADF imaging and electron diffraction 


\section{References:}

[1] Gludovatz, B. et al. Science 345, 1153-1158 (2014).

[2] Gali, A. \& George, E. P. Intermetallics 39, 74-78 (2013).

[3] P. Voyles, J. Grazul, and D. Muller, Ultramicroscopy 96, 251 (2003).

[4] This work is supported by ARO under contract W911NF-14-0285

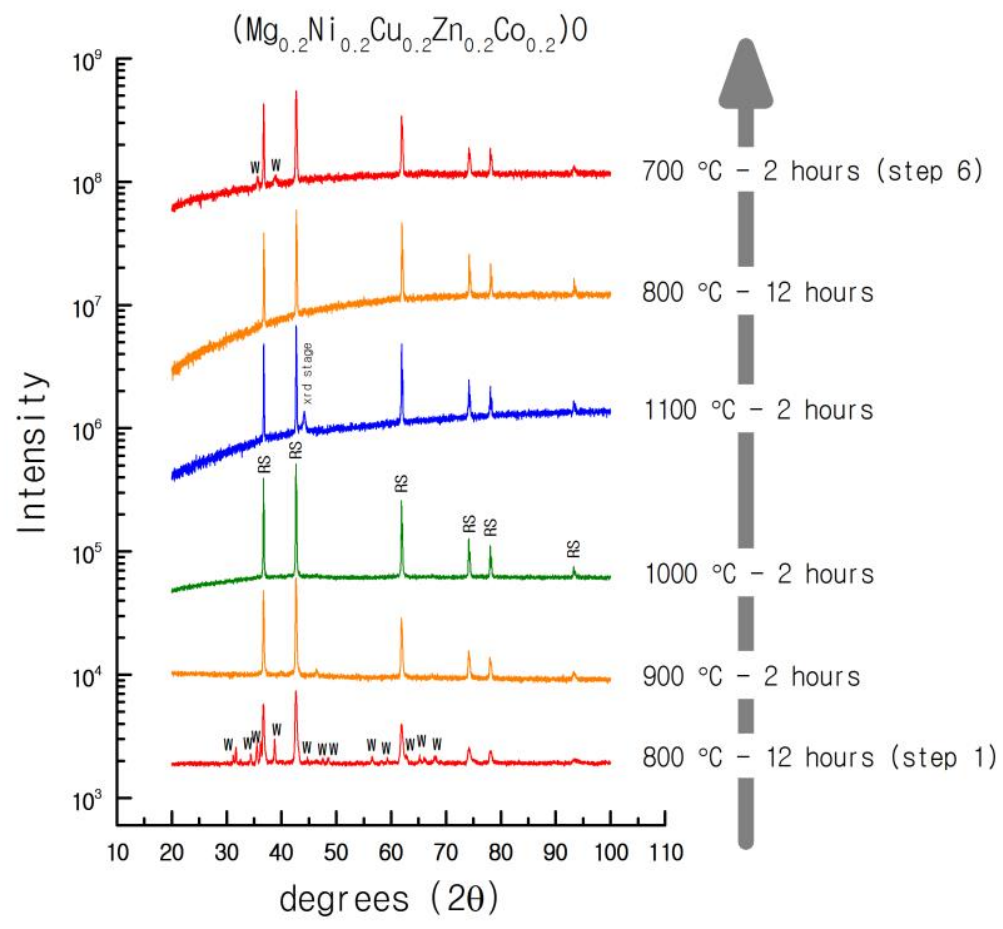

Figure 1. X-ray diffraction patterns of a single pellet during the annealing process. The temperature spanned a range from $700^{\circ} \mathrm{C}$ to $1100^{\circ} \mathrm{C}$, in $50^{\circ} \mathrm{C}$ increments.
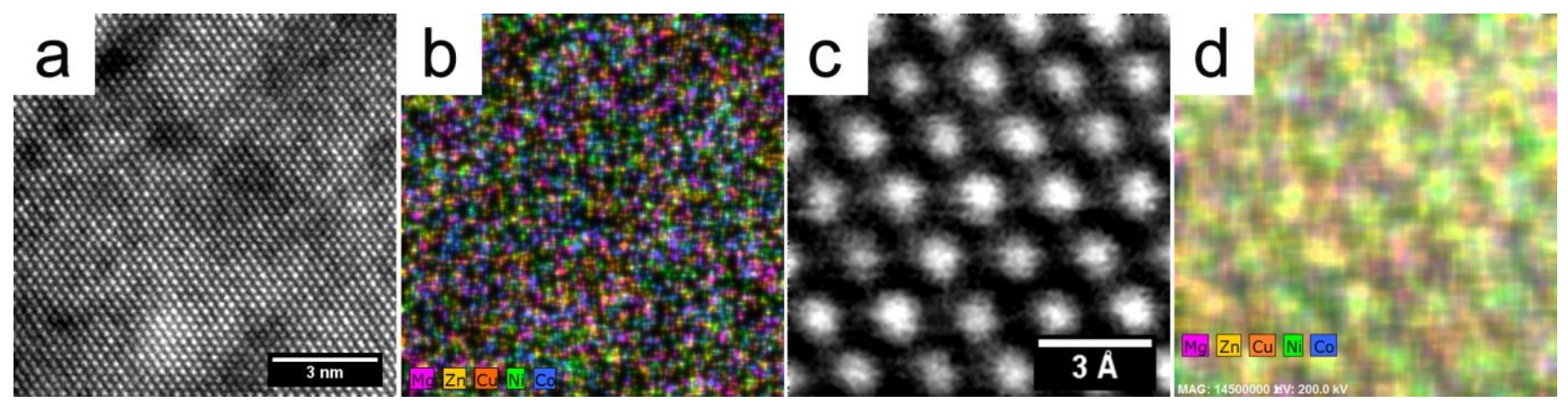

Figure 2. (a) Low magnification HAADF image of single phase rocksalt. (b) EDS map indicates uniform distribution of $\mathrm{Zn}, \mathrm{Ni}, \mathrm{Cu}, \mathrm{Mg}$ and $\mathrm{Co}$. (c) HAADF image at higher magnification taken along <110> direction and (d) EDS map at higher magnification shows distribution of $\mathrm{Zn}, \mathrm{Ni}, \mathrm{Cu}, \mathrm{Mg}$ and $\mathrm{Co}$ at cation sublattice. 